\title{
A naturalistic longitudinal analysis of post-detoxification outcomes in opioid-dependent patients
}

\author{
JO-HANNA IVERS ${ }^{1}{ }^{\circ}$, LINA ZGAGA ${ }^{1}$, BRION SWEENEY ${ }^{2}$, EAMON KEENAN $^{3,4}$, \\ CATHERINE DARKER ${ }^{1}$, BOBBY P. SMYTH ${ }^{1,3}$ \& JOE BARRY ${ }^{1,3}$ \\ ${ }^{1}$ Department of Public Health and Primary Care, Institute of Population Health, Trinity College Dublin, Dublin, Ireland, \\ ${ }^{2}$ Tara Trust, Edinburgh, UK, ${ }^{3}$ National Drug Treatment Centre, Health Service Executive, Dublin, Ireland, and ${ }^{4}$ National \\ Office of Social Inclusion, Health Service Executive, Dublin, Ireland
}

\begin{abstract}
Introduction and Aims. To provide an assessment of outcomes in a cohort of opioid-dependent patients post-detoxification. Design and Methods. This study employed an observational longitudinal cohort design. Patients who completed detoxification in the three major Drug Dependency Units in Ireland during a 14-month period were included in the study $(n=143)$. Patients opting for one of the three pathways post-detoxification (inpatient aftercare, outpatient aftercare or no formal aftercare) were assessed in the final week of detoxification and followed up after 3, 6 and 9 months. The primary outcome was abstinence following detoxification. Results. A Cox (adjusted) model indicated participants who opted for outpatient aftercare treatment lapsed/relapsed at a rate of 52\% higher than the inpatient aftercare group (hazard ratio $=1.52,95 \%$ confidence interval 0.75-3.08, $P=0.24$ ). Moreover, time to lapse/relapse was considerably shorter for the no formal aftercare group (hazard ratio $=7.68,95 \%$ confidence interval 4.30-13.73, $P=5.75 \times 10^{-12}$ ). Abstinence rates for outpatient aftercare and inpatient aftercare are about equal after 9 months. Discussion and Conclusion. Patients who opt for aftercare post-detoxification have significantly better outcomes at follow up when compared to no formal aftercare. In addition, patients' intention to attend aftercare affected their outcomes regardless of eventual treatment path. [Ivers J-H, Zgaga L, Sweeney B, Keenan E, Darker C, Smyth BP, Barry J. A naturalistic longitudinal analysis of post-detoxification outcomes in opioid-dependent patients. Drug Alcohol Rev 2018;37:S339-S347]
\end{abstract}

Key words: addiction, aftercare, detoxification, longitudinal, opioid dependent.

\section{Introduction}

Opioid dependence is a serious public health concern both nationally [1] and internationally [2]. The adverse impacts of this for patients are well established [3-5]. Treatment for opioid dependence is beneficial at an individual [6], family [7] and community level [8]. Ireland has developed a comprehensive response to opioid use over the past 20 years. However, this response has focused almost exclusively on the harm reduction model whereby opioid-dependent patients receive substitution treatment on demand. There are, currently, in excess of 10000 patients receiving methadone maintenance in Ireland. The vast majority of patients attending detoxification in Ireland are receiving methadone. While this has been hugely beneficial in public health terms (i.e. reducing drug-related mortality, transmission of blood borne viruses, in particular HIV, as well as a reduction in drug-related crime) it has limited effect on individuals who wish to become drug-free [9]. Given the weak evidence base for detoxification coupled with the increased risk of overdose post-detoxification and limited capacity in public health system [10], many clinicians opt to continue substitute prescribing or indeed may dissuade opioiddependent persons from detoxifying. In the absence of national statistics for individuals attending detoxification programs in Ireland, the researcher drew data

Jo-Hanna Ivers MA, MSc, PhD, Research Fellow, Lina Zgaga MD, MSc, PhD, Associate Professor, Brion Sweeney MD, Consultant Psychiatrist, Eamon Keenan MD, Consultant Psychiatrist, Catherine Darker MA, PhD, Assistant Professor, Bobby P. Smyth MD, Consultant Psychiatrist, Joe Barry MD, Professor. Correspondence to Dr Jo-Hanna Ivers, Institute of Population Health, Department of Public Health \& Primary Care, Russell Centre, Tallaght Cross West, Tallaght, Dublin 24, Ireland. Tel: +0035318968507; E-mail: jivers@tcd.ie 
from four of the five major detoxification units for the five years preceding this study. On average $160-180$ patients in Ireland attend detoxification programs annually, which is quite small and equates to less than $2 \%$ of the population of patients receiving methadone. The majority of addiction treatment in Ireland occurs within the public health care system, thus access to detoxification is limited in the public domain, creating a blockage for those who need medically supervised detoxification. There is a dearth of data on waiting times for detoxification in Ireland. Available data on waiting times for residential treatment, which includes alcohol detoxification, indicates inadequate level of service provision [11]. A Report of the Health Service Executive Working Group on Residential Treatment \& Rehabilitation (Substance Users) found the provision of beds available for medical detoxification in major deficit. At the time of the report, there were 17.5 inpatient unit beds and the Working Group recommended an increase of 356.5 beds (104 inpatient [detoxification and stabilisation] unit and 252.5 rehabilitation) [11].

One reason for the high relapse rates postdetoxification is perhaps the erroneous view that detoxification is a stand-alone treatment [12]. The primary function of aftercare programs is on preventing relapse and preserving the positive changes created during the detoxification program [13]. Examining the difference between patients who engage in structured aftercare with patients who have no formal aftercare is crucial to treatment development and positive relapse prevention [14]. Attention has shifted to attempting to understand better what characteristics determine patients' choice to attend aftercare [15-17]. The significant improvement in outcomes from undertaking aftercare post-detoxification has been demonstrated for both alcohol and illicit drug users [18]. In addition, Tuten et al. found opioid-dependent patients who entered aftercare post-detoxification had significantly better abstinence rates than patients without aftercare [19]. Furthermore, Tuten et al. [19] found that access to individual counselling prior to detoxification improved aftercare enrolment post-detoxification. Characteristics such as age, gender and previous attempts at treatment appear to be associated with motivation to attend aftercare [20]. Improving rates of transition from detoxification to aftercare is a vital goal for treatment providers. Efforts to achieve this goal should include development and evaluation of specific treatment paths, as well as interventions that enhance treatment transition [21].

Previous international cohort studies of problem drug users in treatment, such as the drug abuse treatment outcome studies [16], Treatment Outcome Prospective Study [22] and National Treatment Outcome Research Study [23], capture a longitudinal picture of drug abuse patterns and treatment responses and have become quite dated. Most studies have been carried out in the USA. Likewise, the data for the National Treatment Outcome Research Study which was carried out by the National Addiction Centre between 1995 and 2000 - the largest UK drug treatment outcome study to date-and The Research Outcome Study in Ireland Evaluating Drug Treatment Effectiveness which was the first national, longitudinal drug treatment outcome study in Ireland, are at least a decade old. In the majority of these cases, the studies have reported solely on the percentage of patients who opted for abstinence programs. Significantly better treatment outcomes are consistently associated with time spent in treatment over fewer episodes. Nevertheless, current data on post-detoxification outcomes are limited, with even less information on factors influencing outcomes for this patient group [16,22,23]. The Australian Treatment Outcome Study (ATOS) included a cohort of 615 patients, the purpose of which was to describe and compare the characteristics of first time heroindependent patients seeking treatment versus nontreatment seeking heroin-dependent patients [17]. The ATOS included but did not focus solely on detoxification. The broader ATOS cohort showed discernible reductions in heroin use by 3 months, and current abstinence continued in more than $50 \%$ of the population at follow up. Continual heroin abstinence was attained by $14 \%$ of ATOS participants within the first 12 months and subsequent analyses revealed that almost a fifth (18\%) of those in the Residential Rehabilitation arm of the study maintained abstinence across 24 months. Furthermore, follow-up studies of this cohort at 12 [24] and 36 [25] months found significant decrease in criminality, psychopathology and injectionrelated health problems following treatment exposure.

Although many international studies suggest they are longitudinal, data are often limited to two timepoints and fail to take into account longitudinal changes [25]. More importantly none of these studies focused on aftercare post-detoxification. Naturalistic prospective longitudinal, multi-site outcome studies of heroin dependence are rare. These studies allow for the examination of the impact of treatment over time, as it is delivered in 'real-world' settings [25].

The overall objective of this study was to investigate naturalistic longitudinal aftercare pathways for patients post-detoxification.

\section{Methods}

Setting

Prior to admission to detoxification in each of the programs, all patients are expected to have commenced 
therapeutic work with an addiction counsellor (minimum of three consecutive sessions, usually bi-monthly) in a community-based treatment service. Additionally, all patients undergo a thorough assessment to examine such areas as psychiatric co-morbidity and motivation to detoxify. The standard treatment program lasts between 4 and 6 weeks. This includes a 10 day methadone detoxification, and a benzodiazepine detoxification if required. Patients receive nursing, counselling and medical support throughout the duration of treatment. During treatment, patients are involved in individual therapy and group therapy. Participants are monitored by their usual key worker/case manager throughout the course of this study.

The three largest residential publicly funded drug dependency units in Ireland acted as a research site. Research site $\mathrm{A}$ is a detoxification unit based in a hospital setting. It is based on a traditional medical model and staffed by a full complement of medical staff. Each patient is under the care of a consultant psychiatrist. Research site $\mathrm{B}$ is a detoxification unit based in a residential setting with two gender-specific units. It incorporates a medically supervised detoxification program (overseen by a general practitioner with a nurse(s) on the premises at all times). Research site C is similar to B in that it is a detoxification unit based in a residential setting that incorporates a medically supervised detoxification program (overseen by a general practitioner with a nurse on premises at all times), however the treatment setting is mixed gender. All three detoxification programs are combined with physical work, daily chores, physical activity, psycho-educational group work and one-toone counselling.

Post-detoxification patients go on one of three pathways; inpatient rehabilitation, outpatient rehabilitation or no formal aftercare. Inpatient aftercare programs typically last between 8 and 16 weeks. Two inpatient facilities were utilised by participants. Inpatient facilities mainly differed on length of program and therapeutic model. All inpatient programs are combined with physical work, daily chores, physical activity, psycho-educational group work and one-toone counselling. Inpatient facilities are overseen by a medical professional. Outpatient facilities typically last between 12 and 24 weeks. Five different outpatient facilities were utilised by participants. The therapeutic model was quite similar for all but one. The program focus typically took either an educational or vocational approach. As part of the recovery process all participants are encouraged to obtain and link with a general practitioner to address their ongoing health needs.

This study was reviewed and approved by the Ethical Committee of the National Drug Treatment Board.

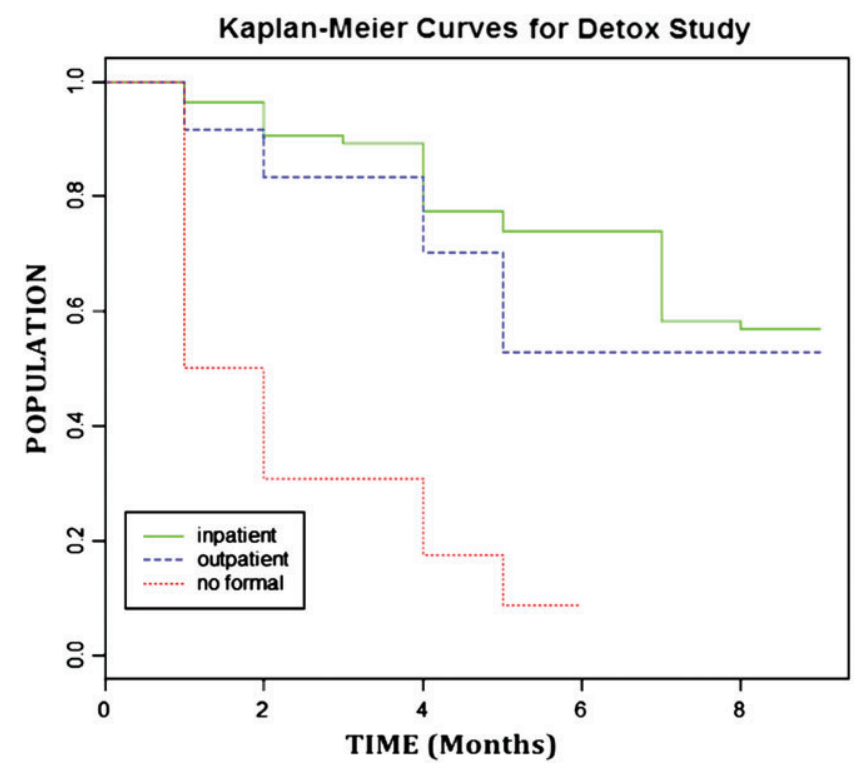

Figure 1. Kaplan-Meier survival curve (unadjusted).

Participants

All opioid-dependent patients admitted to research site $\mathrm{A}, \mathrm{B}$ and $\mathrm{C}$ who had completed the detoxification were eligible to participate in the current study. As the study was examining outcomes post-detoxification, only patients who completed detoxification were screened at baseline (final week of detoxification) and followed up at 3, 6 and 9 month. Patients nominated their chosen rehabilitation path at baseline.

\section{Inclusion criteria}

All patients aged between 18 and 65 years, admitted to the three Drug Dependency Units within the stated timeframe, who had detoxified from opioids between September 2012 and November 2013 were invited to participate in the study. All patients who underwent detoxification and agreed to participate in the study were included in the study. Two patients refused to take part in the study from the outset, as they did not wish to be tracked. Neither of these patients differed from the main population.

\section{Exclusion criteria}

Patients with terminal illness or acute severe psychosis were excluded from participating in the study.

\section{Consent}

Due to the low level of literacy in this population, all potential participants were provided with a Participant 
Information leaflet about the study. The content was also read out and explained to them during an introductory session. If they agreed to participate, participants provided written consent.

All participants were asked to complete a questionnaire that included personal and clinical demographics as well as history of treatment. Prior to data collection, a group of addiction specialists within the addiction services (two consultant psychiatrists, a senior clinical psychologist, a manager of an inpatient rehabilitation unit and a manager of an outpatient community-based rehabilitation program) were contacted and asked to review the demographic questionnaire. Following refinement of demographic questionnaire they were piloted on the first five patients at baseline and at 3 month follow up. No amendments to the demographic questionnaire were necessary following the pilot.

\section{Statistical plan}

As the current study employed a naturalistic longitudinal cohort design, which involved screening and tracking a cohort of patients to observe specific outcomes of interest (abstinence at 3, 6 and 9 month follow up), no attempt was made to influence the patient's treatment path [26].

The effects of treatment path were explored in a longitudinal analysis. This statistical technique allows for exploring correlations among longitudinal observations within and between subjects and for exploring the effects of covariates of interest. $R$ version 3.0.0 statistical software was used for this analysis. The primary outcome was the effect of treatment path on abstinence post-detoxification. We were interested in detecting time to first use postdetoxification, that is, does time to use differ between chosen treatment paths (i.e. inpatient, outpatient or no formal aftercare). In order to do so, we performed an unadjusted Kaplan-Meier curve and a Cox survival analysis on data (Figure 1) and a Cox survival analysis (Table 1) on data. Survival analysis is concerned with studying the time between entry to a study and a subsequent event (use). We applied a Cox proportional hazards regression model as it allowed for testing differences in survival times across

Table 1. Illustrates cox model

\begin{tabular}{|c|c|c|c|c|}
\hline & Estimate & HR & $\begin{array}{l}\text { Std. } \\
\text { error }\end{array}$ & $P$ value \\
\hline Age & -0.01062 & 0.99 & 0.02333 & 0.65 \\
\hline Gender & 0.51200 & 1.67 & 0.26106 & $0.05^{*}$ \\
\hline $\begin{array}{l}\text { As. factor } \\
\text { (outpatient) }\end{array}$ & 0.41900 & 1.52 & 0.36010 & 0.24 \\
\hline $\begin{array}{l}\text { As. factor (no formal } \\
\text { aftercare) }\end{array}$ & 2.03893 & 7.68 & 0.29611 & $5.74 \mathrm{e}-12 * * *$ \\
\hline
\end{tabular}

Both gender* and no formal treatment path ${ }^{* * *}$ were significant. groups, while allowing to adjust for covariates of interest (i.e. age, gender, parenthood, preparatory work, [(Adjunct to compulsory counselling sessions. Varied from individual work with a keyworker/counsellor to engagement in structured preparation program)] previous attempts at detoxification, previous attempts at rehabilitation). The main independent variable(s) were effects of actual treatment path (inpatient, outpatient or no formal aftercare), on abstinence rates at 3, 6 and 9 month follow up. In addition to primary outcome of abstinence rates, the study was interested in examining whether a break in abstinence resulted in lapse (single event) or relapse (return to daily use). Thus, at each follow up, patients were asked if they had used any substance since last follow up (Table 2). The month of use, substance used and whether this was a lapse or relapse was recorded. Patients who could not be followed up are excluded from analysis. In addition, we also examined participants' intended treatment path versus their actual treatment path. The purpose of which was to examine whether intention to go on particular path influenced outcome.

Descriptive statistics were utilised to examine patient characteristics, clinical demographics, as well as factors such as and inpatient facility composition.

\section{Follow-up methods}

Once patients consented to participate in the study, they were asked to provide their contact details and nominate a next of kin, the agencies that they expect to be in contact with and their general practitioner, all of whom could be used as a point of follow up. In addition, patients consented for the researcher to have access to their clinical records via the Central Treatment List, which is a complete register of all patients receiving methadone (as treatment for problem opioid use) in Ireland. The majority of patients $(n=140 /$ $98 \%$ ) were contactable personally or through their nominated agencies. Follow-up questionnaires and interviews were completed in community-based addiction services. In the case of one interviewee not linked with any community service, one interview was conducted in an office on the Trinity College campus.

\section{Results}

One hundred and forty-three patients were recruited into the study across three research sites. Table 3 illustrates clinical profile at baseline across the three treatment pathways.

Table 2 below illustrates the abstinence rates at 3, 6 and 9 month follow up. Abstinence is reported in 
Table 2. Abstinence rates at 3, 6 and 9 month follow up

Abstinence by treatment path at 3 month follow up

\begin{tabular}{|c|c|c|c|c|}
\hline & Yes & No & Could not be followed & Total \\
\hline Inpatient & $73(86 \%)^{\mathrm{a}}$ & 7 (L5/R2) (8\%) & $5(6 \%)$ & 85 \\
\hline Outpatient & $22(85 \%)$ & $4(\mathrm{~L} 1 / \mathrm{R} 3)(15 \%)$ & 0 & 26 \\
\hline Total & $104(73 \%)^{\mathrm{b}}$ & $28(19 \%)$ & $11(8 \%)$ & 143 \\
\hline \multicolumn{5}{|c|}{ Abstinence by treatment path at 6 month follow up } \\
\hline & Yes & No & Could not be followed & Total \\
\hline Inpatient & $64(75 \%)$ & 16 (L6/R10) (19\%) & $5(6 \%)$ & 85 \\
\hline Outpatient & $14(53 \%)$ & $9(\mathrm{~L} 4 / \mathrm{R} 5)(35 \%)$ & $3(12 \%)$ & 26 \\
\hline No formal aftercare & $5(16 \%)$ & 13 (L4/R9) (40\%) & $14(44 \%)$ & 32 \\
\hline Total & $83(58 \%)$ & $38(27 \%)$ & $22(15 \%)$ & 143 \\
\hline
\end{tabular}

Abstinence by treatment path at 9 month follow up

\begin{tabular}{lccr}
\hline & Yes & No & Could not be followed \\
\hline Inpatient & $57(67 \%)$ & $22(\mathrm{~L} 13 / \mathrm{R} 9)(26 \%)$ & $6(7 \%)$ \\
Outpatient & $13(50 \%)$ & $5(\mathrm{~L} 3 / \mathrm{R} 2)(19 \%)$ & $8(31 \%)$ \\
No formal aftercare & $2(6 \%)$ & $8(\mathrm{~L} 2 / \mathrm{R} 6)(25 \%)$ & $22(69 \%)$ \\
Total & $72(50 \%)$ & $35(24 \%)$ & $36(25 \%)$ \\
\end{tabular}

${ }^{a}$ Percentage is taken from available population as per treatment path not entire study population $(n=143) .{ }^{b}$ Percentages for total are taken from entire study population. L, lapse; R, relapse.

Table 3. Clinical demographics of population at baseline broken down by actual treatment path

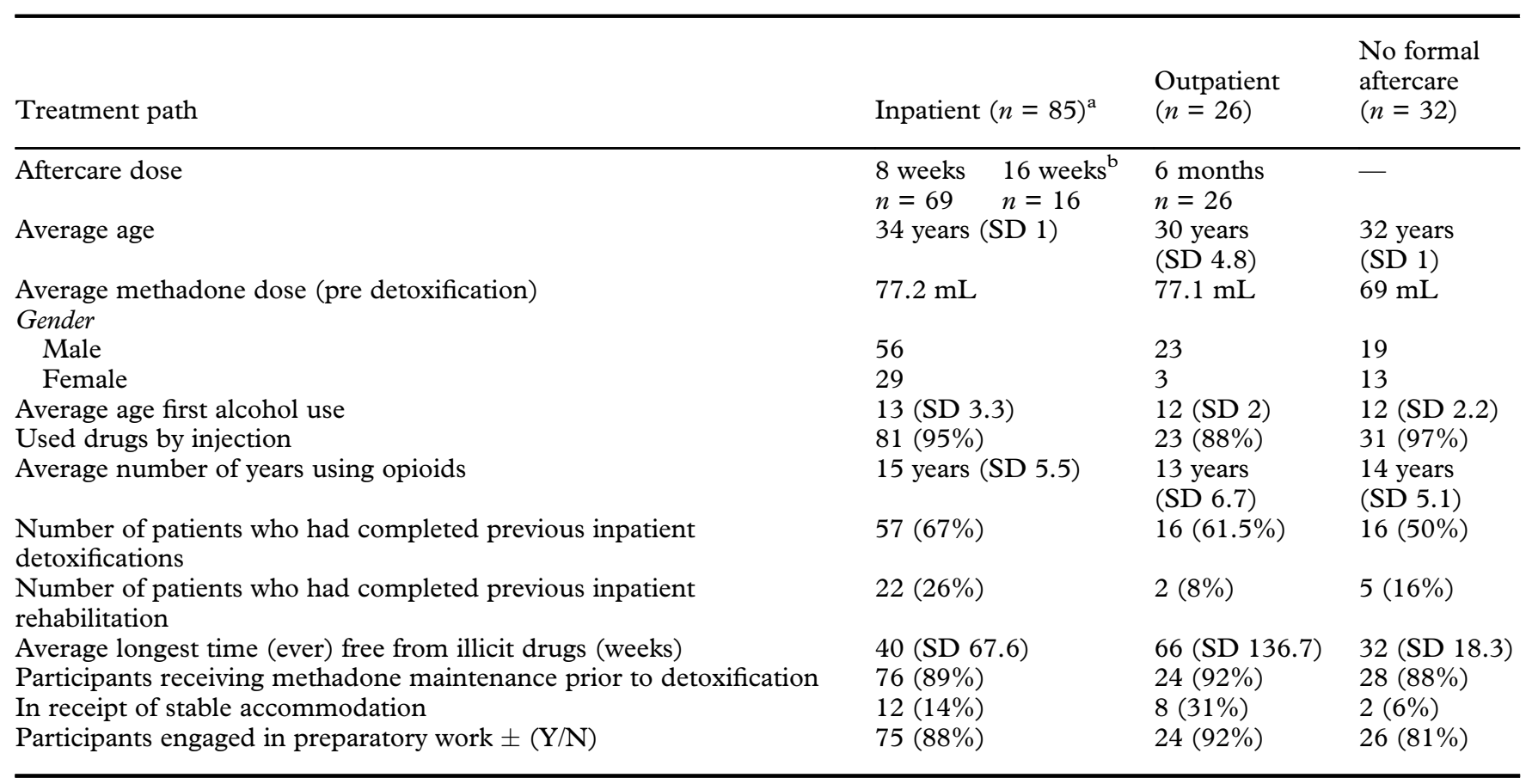

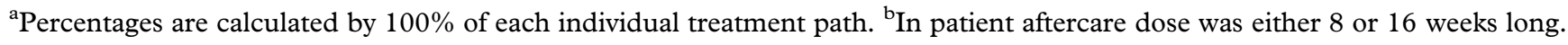


Table 4. First substance used at time of lapse/relapse

\begin{tabular}{|c|c|c|c|c|c|c|c|}
\hline & \multicolumn{6}{|c|}{ Substance } & \multirow[b]{2}{*}{ Total } \\
\hline & Heroin & Alcohol & Benzodiazepines & Cocaine & Cannabis & N/A & \\
\hline Inpatient & $18(13 \%)$ & $8(6 \%)$ & 0 & 1 & $9(6 \%)$ & $49(34 \%)$ & 85 \\
\hline Outpatient & $10(7 \%)$ & 0 & $1(1 \%)$ & 0 & $1(1 \%)$ & $14(10 \%)$ & 26 \\
\hline No formal aftercare & $15(10 \%)$ & $5(3 \%)$ & 0 & 0 & $3(2 \%)$ & $9(6 \%)$ & 32 \\
\hline Total & $43(30 \%)$ & $13(9 \%)$ & $1(1 \%)$ & $1(1 \%)$ & $13(9 \%)$ & $72(50 \%)$ & 143 \\
\hline
\end{tabular}

N/A, not applicable.

terms of self-reported lapse/relapse to any substance since last follow up. Table 4 illustrates substances used. In addition, Table 2 offers a breakdown of lapse (single use) versus relapse (return to daily use) across three treatment pathways. At 3 months, 92\% $(n=132)$ of the population were followed up. Patients who opted for no formal aftercare were most likely to have used drugs. Additionally, patients who opted for either outpatient or no formal aftercare were more likely to have relapsed (returned to daily use) than lapsed (single use). At 6 months, $85 \%(n=121)$ of the population were followed up. Almost one-third of the overall population $(31 \% / n=38)$ reported using a substance at 6 month follow up. The incidence of reported use at 6 month follow up in the inpatient group had more than doubled ( $n=7 \mathrm{~T} 1 / n=16 \mathrm{~T} 2$ ). Consistent with earlier follow up, the inpatient group were more likely to have lapsed rather than relapsed (returned to daily use). At 9 months, $75 \%$ ( $n=107)$ of the population were followed up. The differences in lapse/relapse for no aftercare versus inpatient and outpatient are likely to be higher than reported in the results considering the overrepresentation of drop out in the no formal aftercare group who are quite likely to have experienced a lapse/relapse.

Two inpatient facilities were utilised by participants. The range in treatment time was quite varied between 8 and 16 weeks. A cross-sectional analysis revealed no significant difference in abstinence rates by treatment facility at $6\left(X^{2}=3.3, \mathrm{df}=2, P=0.19\right)$ or $9\left(X^{2}=4.9\right.$, $\mathrm{df}=2, P=0.83$ ) months follow up. The number of previous attempts at inpatient treatment differed considerably across current treatment paths, however, a cross sectional analysis revealed no significant difference in current treatment patient and number of previous treatment attempts $\left(X^{2}=2.8, \mathrm{df}=2, P=0.59\right)$.

\section{Longitudinal data analysis}

The effects of treatment path were explored in a longitudinal analysis. Given that some individuals may not have gone on treatment path proposed at baseline, an analysis comparing both intention (at baseline) to attend inpatient, with no-intention to attend inpatient was preformed, we found that non-intenders yielded two times increased risk for relapse $(P=0.00004)$.

The Kaplan-Meier curve illustrates that $50 \%$ of participants in the no formal aftercare group relapsed within the first month. By month 3, 69\% of the no formal aftercare group had relapsed when compared to $17 \%$ of the outpatient and $11 \%$ of the inpatient. By month $5,30 \%$ of the outpatient group had relapsed; however, the inpatient group relapse rate was below this until month 7 , when it reached $41 \%$. Very few patients with any treatment (inpatient or outpatient) had relapsed in the final 2 months of follow up.

Employing a Cox (adjusted) model association between actual treatment path and timing of first use was examined. Patients who opted for outpatient treatment lapsed/relapsed at a rate of $52 \%$ more than inpatient aftercare group, although the difference was not statistically significant (hazard ratio $=1.52,95 \%$ confidence interval $0.75-3.08, P=0.24$ ). However, time to first use was dramatically shorter for those who received no formal aftercare when compared to those who received inpatient aftercare (hazard ratio $=7.68,95 \%$ confidence interval $4.30-13.73, P=5.75 \times 10^{-12}$ ) highlighting that any aftercare is significantly better than no aftercare.

Results suggest that fewer patients who enter inpatient rehabilitation relapse are more likely to be abstinent at 3, and 6-month follow up when compared to patients attending outpatient and are more likely rehabilitation or patients with no formal aftercare in place. Furthermore, patients who attended any form of aftercare were more likely than the no formal aftercare group to be abstinent at 9 month follow up. We found a significant association for gender (odds ratio $\mathrm{F}$ Vs. $M .=1.67, P=0.05$ ) with females more likely to relapse than males.

\section{Discussion}

The objective of the current study was to provide a detailed assessment of outcomes in a cohort of opioid- 
dependent patient post-detoxification. As a result, it was possible to compare the impact of aftercare among those who completed detoxification. The study compared a group of opioid-dependent patients who completed inpatient detoxification and subsequently went on one of three treatment pathways: (i) rehabilitation unit inpatient; (ii) those went on to an outpatient rehabilitation program; and (iii) those who opted for no formal aftercare.

The study provides a large national sample that is both demographically and clinically representative of opioid-dependent patients in Ireland. A total of 143 patients were included in the longitudinal study. The attrition rate was low and retention rate was high at $75 \%$ at final follow up. An examination of baseline characteristics between patients retained and lost to follow up suggests a higher proportion of the no formal aftercare group were most likely to be lost to follow up. Higher rates of females attended inpatient aftercare. In addition, those who attended inpatient aftercare were relatively older than those who attended either outpatient or opted for no formal aftercare. Interestingly, patients who currently attended inpatient aftercare reported the highest rates of previous attempts at inpatient aftercare. Other clinical demographics (years of use, IV use, detoxification treatment history, initiation) were comparatively similar across all three groups (see Table 3).

The study found a statistically significant risk for relapse for no formal aftercare groups when compared to those who attended inpatient care. Participants who opted for no formal aftercare were $75 \%$ more likely to relapse. It could be argued that differences in treatment outcome might simply reflect differences in pretreatment drug use [27]. However, all three groups were similar on all measures of drug use at admission (see Table 3).

The intention to attend inpatient aftercare had a statistically significant effect on abstinence. An analysis comparing both 'intention (at baseline) to attend' inpatient, with 'no-intention to attend' inpatient found that non-intenders yielded two times increased risk for relapse.

The current study adds to the existing literature of previous outcome studies (Research Outcome Study in Ireland Evaluating Drug Treatment Effectiveness, drug abuse treatment outcome studies, Treatment Outcome Prospective Study, Australian Treatment Outcome Study) by examining treatment effect and change over time with abstinence being the end result.

Significantly better outcomes were observed amongst those who completed detoxification and went on to aftercare. Thus, it appears doubtful that differences in treatment outcome between completers with aftercare and the no formal aftercare can be accounted for by differences in the initial severity of dependence as defined by dose only. Between half and two-thirds of patients across the three groups completed a previous inpatient detoxification. No statistical difference existed for previous attempts at inpatient detoxification across the three pathways.

We found significant association for gender with females more likely to relapse than males, a finding that is consistent with treatment literature [28], with studies typically suggesting an accelerated progression among women for opioids [29]. In addition, females are more likely than their male counterparts to be in a relationship with another drug user [30-32] and more likely to a co-occurring mental health issue and be a parent [29].

Nonetheless, the superior outcome of those who received aftercare might suggest the advantage which ensues from lengthier treatment duration, a finding shown by Simpson and colleagues [33]. The authors found a correlation between better treatment outcome in long-term methadone maintenance, therapeutic communities and drug-free programs [34]. Moreover, this effect could somewhat reflect the fact that while attending an inpatient rehabilitation program, the individual is 'shielded' from the outside stresses or not available to use drugs, thus relapse is simply delayed [35]. Similarly, selection bias (either self-selection or selection by the inpatient service), of the most motivated patients, needs to be considered. Nevertheless, even at 6 months follow up, the inpatient aftercare group was significantly more likely to be abstinent from opioids than the no formal aftercare group. Importantly at 9 months, engaging in either inpatient or outpatient had a significantly larger effect when compared to no formal aftercare group.

The current study supports the finding that opioiddependent patients can have a lapse (i.e. use on a single occasion) without experiencing a relapse (i.e. return to daily use) $[35,36]$. The study supports the notion of a critical period of high risk for relapse following detoxification [36,37]. Furthermore, given that most patients who engaged in aftercare were more likely to lapse than relapse, these finding suggest how this risk can be managed with appropriate supports, even when lapse occurs.

\section{Conclusion}

This study found that patients who enter aftercare are much less likely to relapse than patients who do not. Currently, aftercare is usually sought late in the final stage of detoxification and current capacity means there is greater demand than available places in 
treatment. The marginal benefit that this study demonstrates for inpatient aftercare, over outpatient aftercare, which is almost as effective and a fraction of the price should be taken into account when planning services. The marginal gain for inpatient aftercare is probably attributable to: (i) the fact that people could not use drugs while in a residential setting and (ii) selection (self-selection and selection by the inpatient service) of the most motivated patients. Nevertheless, increasing access to aftercare services requires addressing multiple factors at an individual, service and broader health system level. It requires the individual patient presenting to detoxification with a clearcare plan and the health system increasing capacity to ensure a continuum of care from detoxification to inpatient rehabilitation aftercare. Thus, several factors need to be reformed and new strategies introduced to further enhance patients' success.

The study is not without its limitations. All data are self-reported and therefore open to bias. Additionally, data were collected at 3 month intervals, and consequently where a patient was reporting lapse/ relapse in month 4, this was not reported for a further 2 months, thus may be subject to recall bias. In the current study, the inpatient group had more than 6 weeks in aftercare, which reinforces the probability of improved outcome in this group. However, as more than two-thirds of the no formal aftercare group were not available to follow up, it is not known if they were abstinent at 9 months. A period of 9 months is too short to evaluate long-term effects. Additional studies with post-detox assessment after 3-5 years would be necessary.

\section{Acknowledgements}

The authors wish to thank the patients for participating and the staff from the detoxification units and across the wider addiction services who gave their time and helped with the recruitment and follow-up data collection.

JI conceived and designed the study, JI acquired the data. JI, BS and EK contributed reagents/materials/ tools and LZ carried out the statistical analysis. JB supervised the study and is the guarantor. JI and JB interpreted the data, and critically revised the manuscript for important intellectual content. JI drafted the manuscript. BS, BS, CD, LZ and EK contributed to the paper.

This research did not receive any specific grant from funding agencies in the public, commercial or not-forprofit sectors.

\section{References}

[1] Darker CD, Sweeney BP, El Hassan HO, Smyth BP, JHH IVERS, Barry JM. Brief interventions are effective in reducing alcohol consumption in opiate-dependent methadone-maintained patients: results from an implementation study. Drug Alcohol Rev 2012;31:348-56.

[2] Kanayama G, Hudson JI, Pope HG. Long-term psychiatric and medical consequences of anabolic-androgenic steroid abuse: a looming public health concern? Drug Alcohol Depend 2008;98:1-12.

[3] Fals-Stewart W. Neurocognitive defects and their impact on substance abuse treatment. J Addict Offender Counsel 1993;13:46-57.

[4] Kosten T, Owens SM. Immunotherapy for the treatment of drug abuse. Pharmacol Ther 2005;108:76-85.

[5] Hall W, Lynskey M, Degenhardt L. Heroin use in Australia: its impact on public health and public order. National Drug and Research Centre, University of New South Wales: National Drug and Alcohol Research Centre, 1999.

[6] De Maeyer J, Vanderplasschen W, Camfield L, Vanheule S, Sabbe B, Broekaert E. A good quality of life under the influence of methadone: a qualitative study among opiate-dependent individuals. Int J Nurs Stud 2011;48:1244-57.

[7] Dawe S, Harnett PH, Rendalls V, Staiger P. Improving family functioning and child outcome in methadone maintained families: the parents under pressure programme. Drug Alcohol Rev 2003;22:299-307.

[8] Jürgens R, Ball A, Verster A. Interventions to reduce HIV transmission related to injecting drug use in prison. Lancet Infect Dis 2009;9:57-66.

[9] Banazadeh N, Kheradmand A, Abedi H. Opiate dependents' experiences of the therapeutic relationship in methadone centers; a qualitative study. Addict Health 2009; $1: 12-8$.

[10] Strang J, McCambridge J, Best D, Beswick T, Bearn J, Rees S, Gossip $M$. Loss of tolerance and overdose mortality after inpatient opiate detoxification: follow up study. BMJ 2003;326:959-60.

[11] Des Corrigan O, Gorman A. Report of the HSE Working Group on Residential Treatment \& Rehabilitation (Substance Abuse). Dublin Health Service Executive 2007.

[12] Broers B, Giner F, Dumont P, Mino A. Inpatient opiate detoxification in Geneva: follow-up at 1 and 6 months. Drug Alcohol Depend 2000;58:85-92.

[13] Io M. Broadening the base of treatment for alcohol problems: report of a study by a Committee of the Institute of Medicine, Division of Mental Health and Behavioral Medicine. Washington DC: National Academy Press, 1990.

[14] Coughey K, Feighan K, Cheney R, Klein G. Retention in an aftercare program for recovering women. Subst Use Misuse 1998;33:917-33.

[15] Castaneda R, Lifshutz H, Galanter M, Medalia A, Franco H. Treatment compliance after detoxification among highly disadvantaged alcoholics. Am J Drug Alcohol Abuse 1992;18:223-34.

[16] Hubbard RL, Craddock SG, Anderson J. Overview of 5-year followup outcomes in the drug abuse treatment outcome studies (DATOS). J Subst Abus Treat 2003;25:125-34.

[17] Ross J, Teesson M, Darke S, Lynskey M, Ali R, Ritter A, Cooke R. The characteristics of heroin users entering treatment: findings from the Australian treatment outcome study (ATOS). Drug Alcohol Rev 2005;24:411-8.

[18] Hubbard RL, Craddock SG, Flynn PM, Anderson J, Etheridge RM. Overview of 1-year follow-up outcomes in the drug abuse treatment outcome study (DATOS). Psychol Addict Behav 1997;11:261.

[19] Tuten M, Jones HE, Lertch EW, Stitzer ML. Aftercare plans of inpatients undergoing detoxification. Am J Drug Alcohol Abuse 2007;33:547-55.

[20] Burdon WM, Dang J, Prendergast ML, Messina NP, Farabee D. Differential effectiveness of residential versus outpatient aftercare for parolees from prison-based therapeutic community treatment programs. Subst Abuse Treat Prev Policy 2007;2:16.

[21] Campbell BK, Tillotson CJ, Choi D et al. Predicting outpatient treatment entry following detoxification for injection drug use: the impact of patient and program factors. J Subst Abus Treat 2010;38(Suppl 1): S87-96.

[22] Hubbard RL, Rachal JV, Craddock SG, Cavanaugh ER. Treatment outcome prospective study (TOPS): client characteristics and behaviors before, during, and after treatment. NIDA Res Monog 1984;51:42-68.

[23] Gossop M, Marsden J, Stewart D, Kidd T. The National Treatment Outcome Research Study (NTORS): 4-5 year follow-up results. Addiction 2003;98:291-303. 
[24] Ross J, Teesson M, Darke S, Lynskey M, Ali R, Ritter A, Cooke R. One year outcomes for heroin dependence: findings from the Australian Treatment Outcome Study (ATOS). Drug Alcohol Depend 2006;83:174-80.

[25] Teesson M, Mills K, Ross J, Darke S, Williamson A, Havard A. The impact of treatment on 3 years' outcome for heroin dependence: findings from the Australian treatment outcome study (ATOS). Addiction 2008;103:80-8.

[26] Mann C. Observational research methods. Research design II: cohort, cross sectional, and case-control studies. Emerg Med J 2003;20:54-60.

[27] Stark MJ. Dropping out of substance abuse treatment: a clinically oriented review. Clin Psychol Rev 1992;12:93-116.

[28] Becker JB, Hu M. Sex differences in drug abuse. Front Neuroendocrinol 2008;29:36-47.

[29] Greenfield SF, Back SE, Lawson K, Brady KT. Substance abuse in women. Psychiatr Clin North Am 2010;33:339-55.

[30] Amaro H, Hardy-Fanta C. Gender relations in addiction and recovery. J Psychoactive Drugs 1995;27:325-37.
[31] Falkin GP, Strauss SM. Social supporters and drug use enablers: a dilemma for women in recovery. Addict Behav 2003;28:141-55.

[32] Leverentz AM. The love of a good man? Romantic relationships as a source of support or hindrance for female ex-offenders. J Res Crime Delinq 2006;43:459-88.

[33] Simpson DD. A conceptual framework for drug treatment process and outcomes. J Subst Abus Treat 2004;27:99-121.

[34] Ghodse AH, Reynolds M, Baldacchino AM et al. Treating an opiatedependent inpatient population: a one-year follow-up study of treatment completers and noncompleters. Addict Behav 2002;27:765-78.

[35] Smyth B, Barry J, Keenan E, Ducray K. Lapse and relapse following inpatient treatment of opiate dependence. Ir Med J 2010;103:176-9.

[36] Gossop M, Stewart D, Browne N, Marsden J. Factors associated with abstinence, lapse or relapse to heroin use after residential treatment: protective effect of coping responses. Addiction 2002;97:1259-67.

[37] Gossop M, Marsden J, Stewart D, Treacy S. Change and stability of change after treatment of drug misuse: 2-year outcomes from the National Treatment Outcome Research Study (UK). Addict Behav 2002;27:155-66. 